\title{
Approximation of a zero point of monotone operators with nonsummable errors
}

\section{Takanori lbaraki*}

Correspondence: ibaraki@ynu.ac.jp Department of Mathematics Education, Yokohama National University, Tokiwadai, Hodogaya-ku, Yokohama, 240-8501, Japan

\begin{abstract}
In this paper, we study an iterative scheme for two different types of resolvents of a monotone operator defined on a Banach space. These resolvents are generalizations of resolvents of a monotone operator in a Hilbert space. We obtain iterative approximations of a zero point of a monotone operator generated by the shrinking projection method with errors in a Banach space. Using our result, we discuss some applications.
\end{abstract}

MSC: 47H05; 47H09; 47J25

Keywords: resolvent; monotone operator; metric projection

\section{Introduction}

Let $H$ be a real Hilbert space and let $A \subset H \times H$ be a maximal monotone operator. Then the zero point problem is to find $u \in H$ such that

$$
0 \in A u \text {. }
$$

Such a $u \in H$ is called a zero point (or a zero) of $A$. The set of zero points of $A$ is denoted by $A^{-1} 0$. This problem is connected with many problems in Nonlinear Analysis and Optimization, that is, convex minimization problems, variational inequality problems, equilibrium problems and so on. A well-known method for solving (1.1) is the proximal point algorithm: $x_{1} \in H$ and

$$
x_{n+1}=J_{r_{n}} x_{n}, \quad n=1,2, \ldots
$$

where $\left.\left\{r_{n}\right\} \subset\right] 0, \infty\left[\right.$ and $J_{r_{n}}=\left(I+r_{n} A\right)^{-1}$. This algorithm was first introduced by Martinet [1]. In 1976, Rockafellar [2] proved that if $\liminf _{n} r_{n}>0$ and $A^{-1} 0 \neq \emptyset$, then the sequence $\left\{x_{n}\right\}$ defined by (1.2) converges weakly to a solution of the zero point problem. Later, many researchers have studied this problem; see [3-9] and others.

On the other hand, Kimura [10] introduced the following iterative scheme for finding a fixed point of nonexpansive mappings by the shrinking projection method with error in a Hilbert space:

(c) 2016 Ibaraki. This article is distributed under the terms of the Creative Commons Attribution 4.0 International License (http://creativecommons.org/licenses/by/4.0/), which permits unrestricted use, distribution, and reproduction in any medium, provided you give appropriate credit to the original author(s) and the source, provide a link to the Creative Commons license, and indicate if changes were made. 
Theorem 1.1 (Kimura [10]) Let $C$ be a bounded closed convex subset of a Hilbert space $H$ with $D=\operatorname{diam} C=\sup _{x, y \in C}\|x-y\|<\infty$, and let $T: C \rightarrow H$ be a nonexpansive mapping having a fixed point. Let $\left\{\epsilon_{n}\right\}$ be a nonnegative real sequence such that $\epsilon_{0}=\lim \sup _{n} \epsilon_{n}<\infty$. For a given point $u \in H$, generate an iterative sequence $\left\{x_{n}\right\}$ as follows: $x_{1} \in C$ such that $\left\|x_{1}-u\right\|<\epsilon_{1}, C_{1}=C$,

$$
\begin{aligned}
& C_{n+1}=\left\{z \in C:\left\|z-T x_{n}\right\| \leq\left\|z-x_{n}\right\|\right\} \cap C_{n}, \\
& x_{n+1} \in C_{n+1} \text { such that }\left\|u-x_{n+1}\right\|^{2} \leq d\left(u, C_{n+1}\right)^{2}+\epsilon_{n+1}^{2}
\end{aligned}
$$

for all $n \in \mathbb{N}$. Then

$$
\limsup _{n \rightarrow \infty}\left\|x_{n}-T x_{n}\right\| \leq 2 \epsilon_{0} .
$$

Further, if $\epsilon_{0}=0$, then $\left\{x_{n}\right\}$ converges strongly to $P_{F(T)} u \in F(T)$.

We remark that the original result of the theorem above deals with a family of nonexpansive mappings, and the shrinking projection method was first introduced by Takahashi $e t$ al. [11]. This result was extended to more general Banach spaces by Kimura [12] (see also Ibaraki and Kimura [13]).

In this paper, we study the shrinking projection method with error introduced by Kimura [10] (see also [12,14]). We obtain an iterative approximation of a zero point of a monotone operator generated by the shrinking projection method with errors in a Banach space. Using our result, we discuss some applications.

\section{Preliminaries}

Let $E$ be a real Banach space with its dual $E^{*}$. The normalized duality mapping $J$ from $E$ into $E^{*}$ is defined by

$$
J x=\left\{x^{*} \in E^{*}:\left\langle x, x^{*}\right\rangle=\|x\|^{2}=\left\|x^{*}\right\|^{2}\right\}
$$

for each $x \in E$. We also know the following properties: see $[15,16]$ for more details.

(1) $J x \neq \emptyset$ for each $x \in E$;

(2) if $E$ is reflexive, then $J$ is surjective;

(3) if $E$ is smooth, then the duality mapping $J$ is single valued.

(4) if $E$ is strictly convex, then $J$ is one-to-one and satisfies that $\left\langle x-y, x^{*}-y^{*}\right\rangle>0$ for each $x, y \in E$ with $x \neq y, x^{*} \in J x$ and $y^{*} \in J y$;

(5) if $E$ is reflexive, smooth, and strictly convex, then the duality mapping $J_{*}: E^{*} \rightarrow E$ is the inverse of $J$, that is, $J_{*}=J^{-1}$;

(6) if $E$ uniformly smooth, then the duality mapping $J$ is uniformly norm to norm continuous on each bounded set of $E$.

Let $E$ be a reflexive and strictly convex Banach space and let $C$ be a nonempty closed convex subset of $E$. It is well known that for each $x \in E$ there exists a unique point $z \in C$ such that $\|x-z\|=\min \{\|x-y\|: y \in C\}$. Such a point $z$ is denoted by $P_{C} x$ and $P_{C}$ is called the metric projection of $E$ onto $C$. The following result is well known; see, for instance, [16]. 
Lemma 2.1 Let $E$ be a reflexive, smooth, and strictly convex Banach space, let $C$ be a nonempty closed convex subset of $E$, let $P_{C}$ be the metric projection of $E$ onto $C$, let $x \in E$ and let $x_{0} \in C$. Then $x_{0}=P_{C} x$ if and only if

$$
\left\langle x_{0}-y, J\left(x-x_{0}\right)\right\rangle \geq 0
$$

for all $y \in C$.

Let $C$ be a nonempty closed convex subset of a smooth Banach space $E$. A mapping $T: C \rightarrow E$ is said to be of type (P) [17] if

$$
\langle T x-T y, J(x-T x)-J(y-T y)\rangle \geq 0
$$

for each $x, y \in C$. A mapping $T: C \rightarrow E$ is said to be of type (Q) $[17,18]$ if

$$
\langle T x-T y,(J x-J T x)-(J y-J T y)\rangle \geq 0
$$

for each $x, y \in C$. We denote by $F(T)$ the set of fixed points of $T$. A point $p$ in $C$ is said to be an asymptotic fixed point of $T$ if $C$ contains a sequence $\left\{x_{n}\right\}$ such that $x_{n} \rightarrow p$ and $x_{n}-T x_{n} \rightarrow 0$. The set of all asymptotic fixed points of $T$ is denoted by $\hat{F}(T)$. It is clear that if $T: C \rightarrow E$ is of type (P) and $F(T)$ is nonempty, then

$$
\langle T x-p, J(x-T x)| \geq 0
$$

for each $x \in C$ and $p \in F(T)$. Let $E$ be a reflexive, smooth, and strictly convex Banach space and let $C$ be a nonempty closed convex subset of $E$. It is well known that the metric projection $P_{C}$ of $E$ onto $C$ is a mapping of type (P). We also know that if $T: C \rightarrow E$ is of type $(\mathrm{Q})$ and $F(T)$ is nonempty, then

$$
\langle T x-p, J x-J T x\rangle \geq 0
$$

for each $x \in C$ and $p \in F(T)$.

The following results describe the relation between the set of fixed points and that of asymptotic fixed points for each type of mapping.

Lemma 2.2 (Aoyama-Kohsaka-Takahashi [19]) Let E be a smooth Banach space, let $C$ be a nonempty closed convex subset of $E$ and let $T: C \rightarrow E$ be a mapping of type $(P)$. If $F(T)$ is nonempty, then $F(T)$ is closed and convex and $F(T)=\hat{F}(T)$.

Lemma 2.3 (Kohsaka-Takahashi [18]) Let E be a strictly convex Banach space whose norm is uniformly Gâteaux differentiable, let $C$ be a nonempty closed convex subset of $E$ and let $T: C \rightarrow E$ be a mapping of type (Q). If $F(T)$ is nonempty, then $F(T)$ is closed and convex and $F(T)=\hat{F}(T)$.

In 1984, Tsukada [20] proved the following theorem for the metric projections in a Banach space. For the exact definition of Mosco $\operatorname{limit}_{\mathrm{M}} \mathrm{M}-\lim _{n} C_{n}$, see [21]. 
Theorem 2.4 (Tsukada [20]) Let $E$ be a reflexive and strictly convex Banach space and let $\left\{C_{n}\right\}$ be a sequence of nonempty closed convex subsets of $E$. If $C_{0}=\mathrm{M}-\lim _{n} C_{n}$ exists and is nonempty, then for each $x \in E,\left\{P_{C_{n}} x\right\}$ converges weakly to $P_{C_{0}} x$, where $P_{C_{n}}$ is the metric projection of $E$ onto $C_{n}$. Moreover, if $E$ has the Kadec-Klee property, the convergence is in the strong topology.

One of the simplest example of the sequence $\left\{C_{n}\right\}$ satisfying the condition in this theorem above is a decreasing sequence with respect to inclusion; $C_{n+1} \subset C_{n}$ for each $n \in \mathbb{N}$. In this case, $\mathrm{M}-\lim C_{n}=\bigcap_{n=1}^{\infty} C_{n}$ (see $[7,12,21,22]$ for more details).

Let $E$ be a smooth Banach space and consider the following function $V: E \times E \rightarrow \mathbb{R}$ defined by

$$
V(x, y)=\|x\|^{2}-2\langle x, J y\rangle+\|y\|^{2}
$$

for each $x, y \in E$. We know the following properties:

(1) $(\|x\|-\|y\|)^{2} \leq V(x, y) \leq(\|x\|+\|y\|)^{2}$ for each $x, y \in E$;

(2) $V(x, y)+V(y, x)=2\langle x-y, J x-J y\rangle$ for each $x, y \in E$;

(3) $V(x, y)=V(x, z)+V(z, y)+2\langle x-z, J z-J y\rangle$ for each $x, y, z \in E$;

(4) if $E$ is additionally assumed to be strictly convex, then $V(x, y)=0$ if and only if $x=y$.

Lemma 2.5 (Kamimura-Takahashi [23]) Let E be a smooth and uniformly convex Banach space and let $\left\{x_{n}\right\}$ and $\left\{y_{n}\right\}$ be sequences in $E$ such that either $\left\{x_{n}\right\}$ or $\left\{y_{n}\right\}$ is bounded. If $\lim _{n} V\left(x_{n}, y_{n}\right)=0$, then $\lim _{n}\left\|x_{n}-y_{n}\right\|=0$.

The following results show the existence of mappings $\underline{g}_{r}$ and $\bar{g}_{r}$, related to the convex structures of a Banach space $E$. These mappings play important roles in our result.

Theorem 2.6 (Xu [24]) Let $E$ be a Banach space, $r \in] 0, \infty\left[\right.$ and $B_{r}=\{x \in E:\|x\| \leq r\}$. Then

(i) if $E$ is uniformly convex, then there exists a continuous, strictly increasing, and convex function $\underline{g}_{r}:[0,2 r] \rightarrow\left[0, \infty\left[\right.\right.$ with $\underline{g}_{r}(0)=0$ such that

$$
\|\alpha x+(1-\alpha) y\|^{2} \leq \alpha\|x\|^{2}+(1-\alpha)\|y\|^{2}-\alpha(1-\alpha) \underline{g}_{r}(\|x-y\|)
$$

for all $x, y \in B_{r}$ and $\alpha \in[0,1]$;

(ii) if $E$ is uniformly smooth, then there exists a continuous, strictly increasing, and convex function $\bar{g}_{r}:[0,2 r] \rightarrow\left[0, \infty\left[\right.\right.$ with $\bar{g}_{r}(0)=0$ such that

$$
\|\alpha x+(1-\alpha) y\|^{2} \geq \alpha\|x\|^{2}+(1-\alpha)\|y\|^{2}-\alpha(1-\alpha) \bar{g}_{r}(\|x-y\|)
$$

for all $x, y \in B_{r}$ and $\alpha \in[0,1]$.

Theorem 2.7 (Kimura [12]) Let E be a uniformly smooth and uniformly convex Banach space and let $r>0$. Then the function $\underline{g}_{r}$ and $\bar{g}_{r}$ in Theorem 2.6 satisfies

$$
\underline{g}_{r}(\|x-y\|) \leq V(x, y) \leq \bar{g}_{r}(\|x-y\|)
$$

for all $x, y \in B_{r}$. 


\section{Approximation theorem for the resolvents of type (P)}

In this section, we discuss an iterative scheme of resolvents of a monotone operator defined on a Banach space. Let $E$ be a reflexive, smooth, and strictly convex Banach space. An operator $A \subset E \times E^{*}$ with domain $D(A)=\{x \in E: A x \neq \emptyset\}$ and range $R(A)=\bigcup\{A x: x \in$ $D(A)\}$ is said to be monotone if $\left\langle x-y, x^{*}-y^{*}\right\rangle \geq 0$ for any $\left(x, x^{*}\right),\left(y, y^{*}\right) \in A$. A monotone operator $A$ is said to be maximal if $A=B$ whenever $B \subset E \times E^{*}$ is a monotone operator such that $A \subset B$. We denote by $A^{-1} 0$ the set $\{z \in D(A): 0 \in A z\}$.

Let $C$ be a nonempty closed convex subset of $E$, let $r>0$ and let $A \subset E \times E^{*}$ be a monotone operator satisfying

$$
D(A) \subset C \subset R\left(I+r J^{-1} A\right)
$$

for $r>0$. It is well known that if $A$ is maximal monotone operator, then $R\left(I+r J^{-1} A\right)=E$; see [25-27]. Hence, if $A$ is maximal monotone, then (3.1) holds for $C=\overline{D(A)}$. We also know that $\overline{D(A)}$ is convex; see [28]. If $A$ satisfies (3.1) for $r>0$, we can define the resolvent (of type (P)) $P_{r}: C \rightarrow D(A)$ of $A$ by

$$
P_{r} x=\{z \in E: 0 \in J(z-x)+r A z\}
$$

for all $x \in C$. In other words, $P_{r} x=\left(I+r J^{-1} A\right)^{-1} x$ for all $x \in C$. The Yosida approximation $A_{r}: C \rightarrow E^{*}$ is also defined $A_{r} x=J\left(x-P_{r} x\right) / r$ for all $x \in C$. We know the following; see, for instance, $[15,17,19]$ :

(1) $P_{r}$ is mapping of type (P) from $C$ into $D(A)$;

(2) $\left(P_{r} x, A_{r} x\right) \in A$ for all $x \in C$;

(3) $\left\|A_{r} x\right\| \leq|A x|:=\inf \left\{\left\|x^{*}\right\|: x^{*} \in A x\right\}$ for all $x \in D(A)$;

(4) $F\left(P_{r}\right)=A^{-1} 0$.

We obtain an approximation theorem for a zero point of a monotone operator in a smooth and uniformly convex Banach space by using the resolvent of type (P).

Theorem 3.1 Let $E$ be a smooth and uniformly convex Banach space and let $A \subset E \times$ $E^{*}$ be a monotone operator with $A^{-1} 0 \neq \emptyset$. Let $\left\{r_{n}\right\}$ be a positive real sequence such that $\liminf _{n} r_{n}>0$, let $C$ be a nonempty bounded closed convex subset of $E$ satisfying

$$
D(A) \subset C \subset R\left(I+r_{n} J^{-1} A\right)
$$

for all $n \in \mathbb{N}$ and let $r \in] 0, \infty\left[\right.$ such that $C \subset B_{r}$. Let $\left\{\delta_{n}\right\}$ be a nonnegative real sequence and let $\delta_{0}=\lim \sup _{n} \delta_{n}$. For a given point $u \in E$, generate a sequence $\left\{x_{n}\right\}$ by $x_{1}=x \in C$, $C_{1}=C$, and

$$
\begin{aligned}
& y_{n}=P_{r_{n}} x_{n}, \\
& C_{n+1}=\left\{z \in C:\left\langle y_{n}-z, J\left(x_{n}-y_{n}\right)\right| \geq 0\right\} \cap C_{n}, \\
& x_{n+1} \in\left\{z \in C:\|u-z\|^{2} \leq d\left(u, C_{n+1}\right)^{2}+\delta_{n+1}\right\} \cap C_{n+1},
\end{aligned}
$$

for all $n \in \mathbb{N}$. Then

$$
\limsup _{n \rightarrow \infty}\left\|x_{n}-y_{n}\right\| \leq \underline{g}_{r}^{-1}\left(\delta_{0}\right)
$$

Moreover, if $\delta_{0}=0$, then $\left\{x_{n}\right\}$ converges strongly to $P_{A^{-1} 0} u$. 
Proof Since $C_{n}$ includes $A^{-1} 0 \neq \emptyset$ for all $n \in \mathbb{N},\left\{C_{n}\right\}$ is a sequence of nonempty closed convex subsets and, by definition, it is decreasing with respect to inclusion. Let $p_{n}=P_{C_{n}} u$ for all $n \in \mathbb{N}$. Then, by Theorem 2.4 , we see that $\left\{p_{n}\right\}$ converges strongly to $p_{0}=P_{C_{0}} u$, where $C_{0}=\bigcap_{n=1}^{\infty} C_{n}$. Since $x_{n} \in C_{n}$ and $d\left(u, C_{n}\right)=\left\|u-p_{n}\right\|$, we see that

$$
\left\|u-x_{n}\right\|^{2} \leq\left\|u-p_{n}\right\|^{2}+\delta_{n}
$$

for every $n \in \mathbb{N} \backslash\{1\}$. From Theorem 2.6(i), we see that for $\alpha \in] 0,1[$,

$$
\begin{aligned}
\left\|p_{n}-u\right\|^{2} & \leq\left\|\alpha p_{n}+(1-\alpha) x_{n}-u\right\|^{2} \\
& \leq \alpha\left\|p_{n}-u\right\|^{2}+(1-\alpha)\left\|x_{n}-u\right\|^{2}-\alpha(1-\alpha) \underline{g}_{r}\left(\left\|p_{n}-x_{n}\right\|\right)
\end{aligned}
$$

and thus

$$
\alpha \underline{g}_{r}\left(\left\|p_{n}-x_{n}\right\|\right) \leq\left\|x_{n}-u\right\|^{2}-\left\|p_{n}-u\right\|^{2} \leq \delta_{n} .
$$

As $\alpha \rightarrow 1$, we see that $\underline{g}_{r}\left(\left\|p_{n}-x_{n}\right\|\right) \leq \delta_{n}$ and thus $\left\|p_{n}-x_{n}\right\| \leq \underline{g}_{r}^{-1}\left(\delta_{n}\right)$. Using the definition of $p_{n}$, we see that $p_{n+1} \in C_{n+1}$ and thus

$$
\left\langle y_{n}-p_{n+1}, J\left(x_{n}-y_{n}\right)\right\rangle \geq 0,
$$

or equivalently,

$$
\left\langle x_{n}-p_{n+1}, J\left(x_{n}-y_{n}\right)\right\rangle \geq\left\|x_{n}-y_{n}\right\|^{2} .
$$

Hence we obtain

$$
\left\|x_{n}-y_{n}\right\| \leq\left\|x_{n}-p_{n+1}\right\| \leq\left\|x_{n}-p_{n}\right\|+\left\|p_{n}-p_{n+1}\right\| \leq \underline{g}_{r}^{-1}\left(\delta_{n}\right)+\left\|p_{n}-p_{n+1}\right\|
$$

for every $n \in \mathbb{N} \backslash\{1\}$. Since $\lim _{n} p_{n}=p_{0}$ and $\lim \sup _{n} \delta_{n}=\delta_{0}$, we see that

$$
\limsup _{n \rightarrow \infty}\left\|x_{n}-y_{n}\right\| \leq \underline{g}_{r}^{-1}\left(\delta_{0}\right)
$$

For the latter part of the theorem, suppose that $\delta_{0}=0$. Then we see that

$$
\limsup _{n \rightarrow \infty}\left\|x_{n}-y_{n}\right\| \leq \underline{g}_{r}^{-1}(0)=0
$$

and

$$
\limsup _{n \rightarrow \infty} \underline{g}_{r}\left(\left\|x_{n}-p_{n}\right\|\right) \leq \limsup _{n \rightarrow \infty} \delta_{n}=0 .
$$

Therefore, we obtain

$$
\lim _{n \rightarrow \infty}\left\|x_{n}-y_{n}\right\|=0 \quad \text { and } \quad \lim _{n \rightarrow \infty}\left\|x_{n}-p_{n}\right\|=0 .
$$


Hence, we also obtain

$$
\lim _{n \rightarrow \infty} x_{n}=p_{0} \quad \text { and } \quad \lim _{n \rightarrow \infty} y_{n}=p_{0}
$$

So, from

$$
\left\|y_{n}-P_{r_{1}} y_{n}\right\|=r_{1}\left\|A_{r_{1}} y_{n}\right\| \leq r_{1}\left|A y_{n}\right| \leq r_{1}\left\|\frac{J\left(x_{n}-y_{n}\right)}{r_{n}}\right\|=r_{1}\left\|\frac{x_{n}-y_{n}}{r_{n}}\right\| .
$$

and $\liminf _{n} r_{n}>0$, we see that $\lim _{n}\left\|y_{n}-P_{r_{1}} y_{n}\right\|=0$. Then, by Lemma 2.2 and (3.3), we obtain $x_{n} \rightarrow p_{0} \in \hat{F}\left(P_{r_{1}}\right)=F\left(P_{r_{1}}\right)=A^{-1} 0$. Since $A^{-1} 0 \subset C_{0}$, we get $p_{0}=P_{C_{0}} u=P_{A^{-1} 0} u$, which completes the proof.

\section{Approximation theorem for the resolvents of type (Q)}

We next consider an iterative scheme of resolvents of a monotone operator which is different type of Section 3, in a Banach space. Let $C$ be a nonempty closed convex subset of a reflexive, smooth, and strictly convex Banach space $E$, let $r>0$ and let $A \subset E \times E^{*}$ be a monotone operator satisfying

$$
D(A) \subset C \subset J^{-1} R(J+r A)
$$

for $r>0$. It is well known that if $A$ is maximal monotone operator, then $J^{-1} R(J+r A)=E$; see [25-27]. Hence, if $A$ is maximal monotone, then (4.1) holds for $C=\overline{D(A)}$. We also know that $\overline{D(A)}$ is convex; see [28]. If $A$ satisfies (4.1) for $r>0$, then we can define the resolvent (of type (Q)) $Q_{r}: C \rightarrow D(A)$ of $A$ by

$$
Q_{r} x=\{z \in E: J x \in J z+r A z\}
$$

for all $x \in C$. In other words, $Q_{r} x=(J+r A)^{-1} J x$ for all $x \in C$. We know the following; see, for instance, $[17,18]$ :

(1) $Q_{r}$ is mapping of type (Q) from $C$ into $D(A)$;

(2) $\left(J x-J Q_{r} x\right) / r \in A Q_{r} x$ for all $x \in C$;

(3) $F\left(Q_{r}\right)=A^{-1} 0$.

Before our result, we need the following lemma.

Lemma 4.1 Let $E$ be a reflexive, smooth, and strictly convex Banach space, and let $A \subset$ $E \times E^{*}$ be a monotone operator. Let $r>0$ and $C$ be a closed convex subset of $E$ satisfying (4.1) for $r>0$. Then the following holds:

$$
V\left(x, Q_{r} x\right)+V\left(Q_{r} x, x\right) \leq 2 r\left|x-Q_{r} x, x^{*}\right\rangle
$$

for all $\left(x, x^{*}\right) \in A$.

Proof Let $\left(x, x^{*}\right) \in A$. Since $\left(J x-J Q_{r} x\right) / r \in A Q_{r} x$, we see that

$$
0 \leq\left\langle x-Q_{r} x, x^{*}-\frac{J x-J Q_{r} x}{r}\right\rangle
$$




$$
\begin{aligned}
& \left\langle x-Q_{r} x, \frac{J x-J Q_{r} x}{r}\right\rangle \leq\left\langle x-Q_{r} x, x^{*}\right\rangle, \\
& \left\langle x-Q_{r} x, J x-J Q_{r} x\right\rangle \leq r\left|x-Q_{r} x, x^{*}\right\rangle .
\end{aligned}
$$

From the property of $V$, we see that

$$
V\left(x, Q_{r} x\right)+V\left(Q_{r} x, x\right)=2\left\langle x-Q_{r} x, J x-J Q_{r} x\right\rangle \leq 2 r\left|x-Q_{r} x, x^{*}\right\rangle
$$

for all $\left(x, x^{*}\right) \in A$.

We obtain an approximation theorem for a zero point of a monotone operator in a smooth and uniformly convex Banach space by using the resolvent of type (Q).

Theorem 4.2 Let $E$ be a uniformly smooth and uniformly convex Banach space and let $A \subset E \times E^{*}$ be a monotone operator with $A^{-1} 0 \neq \emptyset$. Let $\left\{r_{n}\right\}$ be a positive real numbers such that $\liminf _{n} r_{n}>0$, let $C$ be a nonempty bounded closed convex subset of $E$ satisfying

$$
D(A) \subset C \subset J^{-1} R\left(J+r_{n} A\right)
$$

for all $n \in \mathbb{N}$ and let $r \in] 0, \infty\left[\right.$ such that $C \subset B_{r}$. Let $\left\{\delta_{n}\right\}$ be a nonnegative real sequence and let $\delta_{0}=\limsup _{n} \delta_{n}$. For a given point $u \in E$, generate a sequence $\left\{x_{n}\right\}$ by $x_{1}=x \in C$, $C_{1}=C$, and

$$
\begin{aligned}
& y_{n}=Q_{r_{n}} x_{n}, \\
& C_{n+1}=\left\{z \in C:\left\langle y_{n}-z, J x_{n}-J y_{n}\right\rangle \geq 0\right\} \cap C_{n}, \\
& x_{n+1} \in\left\{z \in C:\|u-z\|^{2} \leq d\left(u, C_{n+1}\right)^{2}+\delta_{n+1}\right\} \cap C_{n+1},
\end{aligned}
$$

for all $n \in \mathbb{N}$. Then

$$
\limsup _{n \rightarrow \infty}\left\|x_{n}-y_{n}\right\| \leq \underline{g}_{r}^{-1}\left(\bar{g}_{r}\left(\underline{g}_{r}^{-1}\left(\delta_{0}\right)\right)\right)
$$

Moreover, if $\delta_{0}=0$, then $\left\{x_{n}\right\}$ converges strongly to $P_{A^{-1} 0} u$.

Proof Since $C_{n}$ includes $A^{-1} 0 \neq \emptyset$ for all $n \in \mathbb{N},\left\{C_{n}\right\}$ is a sequence of nonempty closed convex subsets and, by definition, it is decreasing with respect to inclusion. Let $p_{n}=P_{C_{n}} u$ for all $n \in \mathbb{N}$. Then, by Theorem 2.4, we see that $\left\{p_{n}\right\}$ converges strongly to $p_{0}=P_{C_{0}} u$, where $C_{0}=\bigcap_{n=1}^{\infty} C_{n}$. Since $x_{n} \in C_{n}$ and $d\left(u, C_{n}\right)=\left\|u-p_{n}\right\|$, we see that

$$
\left\|u-x_{n}\right\|^{2} \leq\left\|u-p_{n}\right\|^{2}+\delta_{n}
$$

for every $n \in \mathbb{N} \backslash\{1\}$. From Theorem 2.6(i), we see that for $\alpha \in] 0,1[$,

$$
\begin{aligned}
\left\|p_{n}-u\right\|^{2} & \leq\left\|\alpha p_{n}+(1-\alpha) x_{n}-u\right\|^{2} \\
& \leq \alpha\left\|p_{n}-u\right\|^{2}+(1-\alpha)\left\|x_{n}-u\right\|^{2}-\alpha(1-\alpha) \underline{g}_{r}\left(\left\|p_{n}-x_{n}\right\|\right)
\end{aligned}
$$


and thus

$$
\alpha \underline{g}_{r}\left(\left\|p_{n}-x_{n}\right\|\right) \leq\left\|x_{n}-u\right\|^{2}-\left\|p_{n}-u\right\|^{2} \leq \delta_{n}
$$

As $\alpha \rightarrow 1$, we see that $\underline{g}_{r}\left(\left\|p_{n}-x_{n}\right\|\right) \leq \delta_{n}$ and thus $\left\|p_{n}-x_{n}\right\| \leq \underline{g}_{r}^{-1}\left(\delta_{n}\right)$. Using the definition of $p_{n}$, we see that $p_{n+1} \in C_{n+1}$ and thus

$$
\left\langle y_{n}-p_{n+1}, J x_{n}-J y_{n}\right\rangle \geq 0 .
$$

From the property of the function $V$, we see that

$$
\begin{aligned}
0 & \leq 2\left\langle y_{n}-p_{n+1}, J x_{n}-J y_{n}\right\rangle \\
& =2\left\langle p_{n+1}-y_{n}, J y_{n}-J x_{n}\right\rangle \\
& =V\left(p_{n+1}, x_{n}\right)-V\left(p_{n+1}, y_{n}\right)-V\left(y_{n}, x_{n}\right) \\
& \leq V\left(p_{n+1}, x_{n}\right)-V\left(y_{n}, x_{n}\right) .
\end{aligned}
$$

By Theorem 2.7, we obtain

$$
\begin{aligned}
V\left(y_{n}, x_{n}\right) & \leq V\left(p_{n+1}, x_{n}\right) \\
& =V\left(p_{n+1}, p_{n}\right)+V\left(p_{n}, x_{n}\right)+2\left\langle p_{n+1}-p_{n}, J p_{n}-J x_{n}\right\rangle \\
& \leq V\left(p_{n+1}, p_{n}\right)+\bar{g}_{r}\left(\left\|p_{n}-x_{n}\right\|\right)+2\left\langle p_{n+1}-p_{n}, J p_{n}-J x_{n}\right\rangle \\
& \leq V\left(p_{n+1}, p_{n}\right)+\bar{g}_{r}\left(\underline{g}_{r}^{-1}\left(\delta_{n}\right)\right)+2\left\langle p_{n+1}-p_{n}, J p_{n}-J x_{n}\right\rangle .
\end{aligned}
$$

Since $\lim \sup _{n} \delta_{n}=\delta_{0}$ and $p_{n} \rightarrow p_{0}$, we see that

$$
\limsup _{n \rightarrow \infty} V\left(y_{n}, x_{n}\right) \leq \bar{g}_{r}\left(\underline{g}_{r}^{-1}\left(\delta_{0}\right)\right)
$$

Therefore, by Theorem 2.7, we see that

$$
\limsup _{n \rightarrow \infty}\left\|x_{n}-y_{n}\right\| \leq \limsup _{n \rightarrow \infty} \underline{g}_{r}^{-1}\left(V\left(y_{n}, x_{n}\right)\right) \leq \underline{g}_{r}^{-1}\left(\bar{g}_{r}\left(\underline{g}_{r}^{-1}\left(\delta_{0}\right)\right)\right)
$$

For the latter part of the theorem, suppose that $\delta_{0}=0$. Then we see that

$$
\limsup _{n \rightarrow \infty}\left\|x_{n}-y_{n}\right\| \leq \underline{g}_{r}^{-1}\left(\bar{g}_{r}\left(\underline{g}_{r}^{-1}(0)\right)\right)=0
$$

and

$$
\limsup _{n \rightarrow \infty} \underline{g}_{r}\left(\left\|x_{n}-p_{n}\right\|\right) \leq \limsup _{n \rightarrow \infty} \delta_{n}=0 .
$$

Therefore, we obtain

$$
\lim _{n \rightarrow \infty}\left\|x_{n}-y_{n}\right\|=0 \quad \text { and } \quad \lim _{n \rightarrow \infty}\left\|x_{n}-p_{n}\right\|=0
$$


Hence, we also obtain

$$
\lim _{n \rightarrow \infty} x_{n}=p_{0} \quad \text { and } \quad \lim _{n \rightarrow \infty} y_{n}=p_{0}
$$

Since $E$ is uniformly smooth, the duality mapping $J$ is uniformly norm-to-norm continuous on each bounded subset on $E$. Therefore, we obtain

$$
\lim _{n \rightarrow \infty}\left\|J x_{n}-J y_{n}\right\|=0
$$

From Lemma 4.1 we see that

$$
V\left(y_{n}, Q_{r_{1}} y_{n}\right) \leq V\left(y_{n}, Q_{r_{1}} y_{n}\right)+V\left(Q_{r_{1}} y_{n}, y_{n}\right) \leq 2 r_{1}\left\langle y_{n}-Q_{r_{1}} y_{n}, x^{*}\right\rangle
$$

for all $x^{*} \in A y_{n}$. From $y_{n}, Q_{r_{1}} y_{n} \in D(A) \subset C \subset B_{r}$ and $\left(J x_{n}-J y_{n}\right) / r_{n} \in A y_{n}$, we see that

$$
\begin{aligned}
V\left(y_{n}, Q_{r_{1}} y_{n}\right) & \leq 2 r_{1}\left\langle y_{n}-Q_{r_{1}} y_{n}, \frac{J x_{n}-J y_{n}}{r_{n}}\right\rangle \\
& \leq 2 r_{1}\left\|y_{n}-Q_{r_{1}} y_{n}\right\|\left\|\frac{J x_{n}-J y_{n}}{r_{n}}\right\| \\
& \leq 2 r_{1}\left(\left\|y_{n}\right\|+\left\|Q_{r_{1}} y_{n}\right\|\right)\left\|\frac{J x_{n}-J y_{n}}{r_{n}}\right\| \\
& =4 r_{1} r\left\|\frac{J x_{n}-J y_{n}}{r_{n}}\right\| .
\end{aligned}
$$

Since $\liminf { }_{n} r_{n}>0$ and (4.4), we obtain

$$
\limsup _{n \rightarrow \infty} V\left(y_{n}, Q_{r_{1}} y_{n}\right) \leq 0
$$

This implies $\lim _{n} V\left(y_{n}, Q_{r_{1}} y_{n}\right)=0$. From Theorem 2.5, we see that

$$
\lim _{n \rightarrow \infty}\left\|y_{n}-Q_{r_{1}} y_{n}\right\|=0
$$

Then, by Lemma 2.3 and (4.3), we see that $x_{n} \rightarrow p_{0} \in \hat{F}\left(Q_{r_{1}}\right)=F\left(Q_{r_{1}}\right)=A^{-1} 0$. Since $A^{-1} 0 \subset$ $C_{0}$, we get $p_{0}=P_{C_{0}} u=P_{A^{-1} 0} u$, which completes the proof.

\section{Applications}

In this section, we give some applications of Theorems 3.1 and 4.2. We first study the convex minimization problem: Let $E$ be a reflexive, smooth, and strictly convex Banach space with its dual $E^{*}$ and let $\left.f: E \rightarrow\right]-\infty, \infty$ ] be a proper lower semicontinuous convex function. Then the subdifferential $\partial f$ of $f$ is defined as follows:

$$
\partial f(x)=\left\{x^{*} \in E^{*}: f(x)+\left\langle y-x, x^{*}\right\rangle \leq f(y), \forall y \in E\right\}
$$

for all $x \in E$. By Rockafellar's theorem $[29,30]$, the subdifferential $\partial f \subset E \times E^{*}$ is maximal monotone. It is easy to see that $(\partial f)^{-1} 0=\operatorname{argmin}\{f(x): x \in E\}$. It is also known that, see, for instance, $[15,27,28]$, 


$$
D(\partial f) \subset D(f) \subset \overline{D(\partial f)} .
$$

As a direct consequence of Theorems 3.1 and 4.2, we can show the following corollaries.

Corollary 5.1 Let $E$ be a smooth and uniformly convex Banach space, let $f: E \rightarrow]-\infty, \infty]$ be a proper lower semicontinuous convex function with $D(f)$ being bounded, and let $r \in$ ]0, $\infty\left[\right.$ such that $D(f) \subset B_{r}$. Let $\left\{\delta_{n}\right\}$ be a nonnegative real sequence and let $\delta_{0}=\lim \sup _{n} \delta_{n}$. For a given point $u \in E$, generate a sequence $\left\{x_{n}\right\}$ by $x_{1}=x \in \overline{D(f)}, C_{1}=\overline{D(f)}$, and

$$
\begin{aligned}
& y_{n}=\underset{y \in E}{\operatorname{argmin}}\left\{f(y)+\frac{1}{2 r_{n}}\left\|y-x_{n}\right\|^{2}\right\}, \\
& C_{n+1}=\left\{z \in \overline{D(f)}:\left\langle y_{n}-z, J\left(x_{n}-y_{n}\right)\right\rangle \geq 0\right\} \cap C_{n}, \\
& x_{n+1} \in\left\{z \in \overline{D(f)}:\|u-z\|^{2} \leq d\left(u, C_{n+1}\right)^{2}+\delta_{n+1}\right\} \cap C_{n+1},
\end{aligned}
$$

for all $n \in \mathbb{N}$, where $\left.\left\{r_{n}\right\} \subset\right] 0, \infty\left[\right.$ such that $\liminf _{n} r_{n}>0$. If $(\partial f)^{-1} 0$ is nonempty, then

$$
\limsup _{n \rightarrow \infty}\left\|x_{n}-y_{n}\right\| \leq \underline{g}_{r}^{-1}\left(\delta_{0}\right)
$$

Moreover, if $\delta_{0}=0$, then $\left\{x_{n}\right\}$ converges strongly to $P_{(\partial f)^{-1} 0} u$.

Proof Put $C=\overline{D(f)}$. Since the subdifferential $\partial f \subset E \times E^{*}$ is maximal monotone, we have $E=R(I+r \partial f)$ for all $r>0$ and hence, from (5.1), we see that

$$
D(\partial f) \subset \overline{D(\partial f)}=\overline{D(f)}=C \subset E=R(I+r \partial f)
$$

for all $r>0$.

Fix $r>0$ and $z \in C$. Let $P_{r}$ be the resolvent (of type (P)) of $\partial f$, then we also know that

$$
P_{r} z=\underset{y \in E}{\operatorname{argmin}}\left\{f(y)+\frac{1}{2 r}\|y-z\|^{2}\right\} .
$$

Therefore, we obtain the desired result by Theorem 3.1.

Corollary 5.2 Let E be a uniformly smooth and uniformly convex Banach space, let $f$ : $E \rightarrow]-\infty, \infty]$ be a proper lower semicontinuous convex function with $D(f)$ being bounded and let $r \in] 0, \infty\left[\right.$ such that $D(f) \subset B_{r}$. Let $\left\{\delta_{n}\right\}$ be a nonnegative real sequence and let $\delta_{0}=$ $\lim \sup _{n} \delta_{n}$. For a given point $u \in E$, generate a sequence $\left\{x_{n}\right\}$ by $x_{1}=x \in \overline{D(f)}, C_{1}=\overline{D(f)}$, and

$$
\begin{aligned}
& y_{n}=\underset{y \in E}{\operatorname{argmin}}\left\{f(y)+\frac{1}{2 r_{n}}\|y\|^{2}-\frac{1}{r_{n}}\left\langle y, J x_{n}\right\rangle\right\}, \\
& C_{n+1}=\left\{z \in \overline{D(f)}:\left\langle y_{n}-z, J x_{n}-J y_{n}\right\rangle \geq 0\right\} \cap C_{n}, \\
& x_{n+1} \in\left\{z \in \overline{D(f)}:\|u-z\|^{2} \leq d\left(u, C_{n+1}\right)^{2}+\delta_{n+1}\right\} \cap C_{n+1},
\end{aligned}
$$

for all $n \in \mathbb{N}$, where $\left.\left\{r_{n}\right\} \subset\right] 0, \infty\left[\right.$ such that $\liminf _{n} r_{n}>0$. If $(\partial f)^{-1} 0$ is nonempty, then

$$
\limsup _{n \rightarrow \infty}\left\|x_{n}-y_{n}\right\| \leq \underline{g}_{r}^{-1}\left(\bar{g}_{r}\left(\underline{g}_{r}^{-1}\left(\delta_{0}\right)\right)\right) \text {. }
$$

Moreover, if $\delta_{0}=0$, then $\left\{x_{n}\right\}$ converges strongly to $P_{(\partial f)^{-1} 0} u$. 
Proof Fix $r>0$ and $z \in C$. Let $Q_{r}$ be the resolvent (of type (Q)) of $\partial f$, then we also know that

$$
Q_{r} z=\underset{y \in E}{\operatorname{argmin}}\left\{f(y)+\frac{1}{2 r}\|y\|^{2}-\frac{1}{r}\langle y, J z\rangle\right\}
$$

In the same way as Corollary 5.1, we obtain the desired result by Theorem 4.2.

Next, we study the approximation of fixed points for mappings of type (P) and (Q). Before show our applications, we need the following results.

Lemma 5.3 ([17]) Let E be a reflexive, smooth, and strictly convex Banach space, let $C$ be a nonempty subset of $E$, let $T: C \rightarrow E$ be a mapping, and let $A_{T} \subset E \times E^{*}$ be an operator defined by $A_{T}=J\left(T^{-1}-I\right)$. Then $T$ is of mapping of type $(P)$ if and only if $A_{T}$ is monotone. In this case $T=\left(I+J^{-1} A_{T}\right)^{-1}$.

Lemma 5.4 ([31]) Let E be a reflexive, smooth, and strictly convex Banach space, let $C$ be a nonempty subset of $E$ and let $T: C \rightarrow E$ be a mapping, and let $A_{T} \subset E \times E^{*}$ be an operator defined by $A_{T}=J T^{-1}-J$. Then $T$ is a mapping of type $(Q)$ if and only if $A_{T}$ is monotone. In this case $T=\left(J+A_{T}\right)^{-1} J$.

As a direct consequence of Theorems 3.1 and 4.2, we can show the following corollaries.

Corollary 5.5 Let E be a smooth and uniformly convex Banach space, let $C$ be a bounded closed convex subset of E. Let $T: C \rightarrow C$ be a mapping of type $(P)$ with $F(T)$ being nonempty and let $r \in] 0, \infty\left[\right.$ such that $C \subset B_{r}$. Let $\left\{\delta_{n}\right\}$ be a nonnegative real sequence and let $\delta_{0}=$ $\lim \sup _{n} \delta_{n}$. For a given point $u \in E$, generate a sequence $\left\{x_{n}\right\}$ by $x_{1}=x \in C, C_{1}=C$, and

$$
\begin{aligned}
& C_{n+1}=\left\{z \in C:\left\langle T x_{n}-z, J\left(x_{n}-T x_{n}\right)\right| \geq 0\right\} \cap C_{n}, \\
& x_{n+1} \in\left\{z \in C:\|u-z\|^{2} \leq d\left(u, C_{n+1}\right)^{2}+\delta_{n+1}\right\} \cap C_{n+1},
\end{aligned}
$$

for all $n \in \mathbb{N}$, where $\left\{r_{n}\right\} \subset(0, \infty)$ such that $\liminf _{n} r_{n}>0$. Then

$$
\limsup _{n \rightarrow \infty}\left\|x_{n}-T x_{n}\right\| \leq \underline{g}_{r}^{-1}\left(\delta_{0}\right)
$$

Moreover, if $\delta_{0}=0$, then $\left\{x_{n}\right\}$ converges strongly to $P_{F(T)} u$.

Proof Put $A_{T}=J\left(T^{-1}-I\right)$ and $r_{n}=1$ for all $n \in \mathbb{N}$. From Lemma 5.3, we see that $T$ is the resolvent (of type (P)) of $A_{T}$ for 1 and

$$
D\left(A_{T}\right)=R(T) \subset C=D(T)=R\left(I+J^{-1} A_{T}\right) .
$$

Therefore, we obtain the desired result by Theorem 3.1.

Corollary 5.6 Let E be a uniformly smooth and uniformly convex Banach space, let $C$ be a bounded closed convex subset of E. Let $T: C \rightarrow C$ be a mapping of type $(Q)$ with $F(T)$ being nonempty and let $r \in] 0, \infty\left[\right.$ such that $C \subset B_{r}$. Let $\left\{\delta_{n}\right\}$ be a nonnegative real sequence and 
let $\delta_{0}=\limsup _{n} \delta_{n}$. For a given point $u \in E$, generate a sequence $\left\{x_{n}\right\}$ by $x_{1}=x \in C, C_{1}=C$, and

$$
\begin{aligned}
& C_{n+1}=\left\{z \in C:\left\langle T x_{n}-z, J x_{n}-J T x_{n}\right\rangle \geq 0\right\} \cap C_{n}, \\
& x_{n+1} \in\left\{z \in C:\|u-z\|^{2} \leq d\left(u, C_{n+1}\right)^{2}+\delta_{n+1}\right\} \cap C_{n+1},
\end{aligned}
$$

for all $n \in \mathbb{N}$. Then

$$
\limsup _{n \rightarrow \infty}\left\|x_{n}-T x_{n}\right\| \leq \underline{g}_{r}^{-1}\left(\bar{g}_{r}\left(\underline{g}_{r}^{-1}\left(\delta_{0}\right)\right)\right)
$$

Moreover, if $\delta_{0}=0$, then $\left\{x_{n}\right\}$ converges strongly to $P_{F(T)} u$.

Proof In the same way as Corollary 5.5, we obtain the desired result by Lemma 5.4 and Theorem 4.2 .

\section{Competing interests}

The author declares to have no competing interests.

\section{Acknowledgements}

The author is supported by Grant-in-Aid for Young Scientific (B) No. 24740075 from the Japan Society for the Promotion of Science.

\section{Received: 30 November 2015 Accepted: 29 March 2016 Published online: 11 April 2016}

\section{References}

1. Martinet, B: Régularsation d'inéquations variationnelles par approximations successives. Rev. Francaise Informat. Recherche Opérationnelles 4, 154-158 (1970) (in French)

2. Rockafellar, RT: Monotone operators and proximal point algorithm. SIAM J. Control Optim. 14, 877-898 (1976)

3. Lions, PL: Une méthode itérative de résolution d'une inéquation variationnelle. Isr. J. Math. 31, $204-208$ (1978)

4. Güler, O: On the convergence of the proximal point algorithm for convex minimization. SIAM J. Control Optim. 29, 403-419 (1991)

5. Ibaraki, T: Strong convergence theorems for zero point problems and equilibrium problems in a Banach space. In: Nonlinear Analysis and Convex Analysis I, pp. 115-126. Yokohama Publishers, Yokohama, Japan (2013)

6. Kamimura, S, Takahashi, W: Approximating solutions of maximal monotone operators in Hilbert spaces. J. Approx. Theory 106, 226-240 (2000)

7. Kimura, Y, Takahashi, W: On a hybrid method for a family of relatively nonexpansive mappings in a Banach space. J. Math. Anal. Appl. 357, 356-363 (2009)

8. Ohsawa, S, Takahashi, W: Strong convergence theorems for resolvents of maximal monotone operators in Banach spaces. Arch. Math. 81, 439-445 (2003)

9. Solodov, MV, Svaiter, BF: Forcing strong convergence of proximal point iterations in a Hilbert space. Math. Program. Ser. A 87, 189-202 (2000)

10. Kimura, Y: Approximation of a common fixed point of a finite family of nonexpansive mappings with nonsummable errors in a Hilbert space. J. Nonlinear Convex Anal. 15, 429-436 (2014)

11. Takahashi, W, Takeuchi, Y, Kubota, R: Strong convergence theorems by hybrid methods for families of nonexpansive mappings in Hilbert spaces. J. Math. Anal. Appl. 341, 276-286 (2008)

12. Kimura, Y: Approximation of a fixed point of nonlinear mappings with nonsummable errors in a Banach space. In: Maligranda, L, Kato, M, Suzuki, T (eds.) Proceedings of the International Symposium on Banach and Function Spaces IV, (Kitakyushu, Japan), pp. 303-311 (2014)

13. Ibaraki, T, Kimura, Y: Approximation of a fixed point of generalized firmly nonexpansive mappings with nonsummable errors. Linear Nonlinear Anal. (to appear)

14. Kimura, Y: Approximation of a fixed point of nonexpansive mapping with nonsummable errors in a geodesic space. In: Proceedings of the 10th International Conference on Fixed Point Theory and Its Applications, pp. 157-164 (2012)

15. Barbu, V: Nonlinear Semigroups and Differential Equations in Banach Spaces. Editura Academiei Republicii Socialiste România, Bucharest (1976)

16. Takahashi, W: Nonlinear Functional Analysis - Fixed Point Theory and Its Applications. Yokohama Publishers, Yokohama, Japan (2000)

17. Aoyama, K, Kohsaka, F, Takahashi, W: Three generalizations of firmly nonexpansive mappings: their relations and continuity properties. J. Nonlinear Convex Anal. 10, 131-147 (2009)

18. Kohsaka, F, Takahashi, W: Existence and approximation of fixed points of firmly nonexpansive type mappings in Banach spaces. SIAM J. Optim. 19, 824-835 (2008)

19. Aoyama, K, Kohsaka, F, Takahashi, W: Strong convergence theorems for a family of mappings of type (P) and applications. In: Proceedings of Asian Conference on Nonlinear Analysis and Optimization, pp. 1-17. Yokohama Publishers, Yokohama, Japan (2009) 
20. Tsukada, M: Convergence of best approximations in a smooth Banach space. J. Approx. Theory 40, $301-309$ (1984)

21. Mosco, U: Convergence of convex sets and of solutions of variational inequalities. Adv. Math. 3, $510-585$ (1969)

22. Beer, G: Topologies on Closed and Closed Convex Sets. Kluwer Academic, Dordrecht (1993)

23. Kamimura, S, Takahashi, W: Strong convergence of a proximal-type algorithm in a Banach space. SIAM J. Optim. 13 938-945 (2002)

24. Xu, HK: Inequalities in Banach spaces with applications. Nonlinear Anal. 16, 1127-1138 (1991)

25. Browder, FE: Nonlinear maximal monotone operators in Banach space. Math. Ann. 175, 89-113 (1968)

26. Rockafellar, RT: On the maximality of sums of nonlinear monotone operators. Trans. Am. Math. Soc. 149, 75-88 (1970)

27. Takahashi, W: Convex Analysis and Approximation of Fixed Points. Yokohama Publishers, Yokohama, Japan (2000) (in Japanese)

28. Rockafellar, RT: On the virtual convexity of the domain and range of a nonlinear maximal monotone operator. Math. Ann. 185, 81-90 (1970)

29. Rockafellar, RT: Characterization of the subdifferentials of convex functions. Pac. J. Math. 17, 497-510 (1966)

30. Rockafellar, RT: On the maximal monotonicity of subdifferential mappings. Pac. J. Math. 33, $209-216$ (1970)

31. Kohsaka, F, Takahashi, W: Fixed point theorems for a class of nonlinear mappings related to maximal monotone operators in Banach spaces. Arch. Math. (Basel) 91, 166-177 (2008)

\section{Submit your manuscript to a SpringerOpen ${ }^{\ominus}$ journal and benefit from:}

- Convenient online submission

- Rigorous peer review

- Immediate publication on acceptance

- Open access: articles freely available online

- High visibility within the field

- Retaining the copyright to your article 